\title{
Rauheitsmessung an pharmazeutischen Tabletten mittels Angularer Speckle-Korrelation
}

\author{
Roughness measurement on pharmaceutical tablets using angular speckle correlation
}

Zusammenfassung: Eine vollständige Charakterisierung von pharmazeutischen Tabletten besteht nicht nur in der Analyse der chemischen Zusammensetzung, sondern auch in der Untersuchung der Fertigung der inneren Struktur und der Oberflächenstruktur. Ziel dieser Arbeit ist es, die Oberflächenrauheit von Tabletten an Originalprä paraten und Generika mittels Angularer Speckle Korre lation (ASK) $\mathrm{zu}$ bestimmen. Die Ergebnisse zeigen un terschiedliche Rauheitswerte bei Tabletten mit gleichem Wirkstoff bei verschiedenen Trägerstoffen. Berührungslose Speckle-Rauheitsmessung könnte eingesetzt werden, um die Regelung des Produktionsprozesses zu verbessern, z. B. während die Tabletten aus Pulver gepresst werden.

Schlüsselwörter: Speckle, Rauheit, Tabletten, pharma zeutisch.

Abstract: A complete characterization of pharmaceutical tablets requires not only the analysis of its chemical com pounds, but also of its inner and surface structure manufacturing. The aim of this work is to determine the surface roughness of original and generic tablets by means of angular speckle correlation (ASC). The results show different roughness properties between tablets with the same active ingredients, but with different excipients. Contactless speckle roughness measurements could be used to im prove the control of the production process, e.g., when tablets are pressed from powder.

Keywords: Speckle, roughness, tablets, pharmaceutical.

DOI 10.1515/teme-2014-0403

Eingang 25. November 2013; angenommen 16. März 2014

*Korrespondenzautor: Félix Salazar Bloise, Universidad Politécnica de Madrid, E-Mail: felixjose.salazar@upm.es

Laura Aulbach, Martin Jakobi, Alexander W. Koch: Technische Universität München, Lehrstuhl für Messsystem- und Sensortechnik

\section{Einleitung}

Die Untersuchung der physikalischen Eigenschaften pharmazeutischer Tabletten ist nicht nur für den Verbraucher, sondern auch für die pharmazeutische und medizinische Industrie von großer Bedeutung. In diesem Bereich führt die Herstellung von Materialien, die aus Pulver produziert (gepresst, geklebt) werden, beispielsweise in den USA zu einem Wirtschaftsumsatz von etwa einer Milliarde Dollar jährlich. Mehr als $60 \%$ der pharmazeutischen USIndustriegüter basieren auf solchen Materialien und deren Technologien, und in der Indus-trieproduktion werden $\mathbf{8 0 \%}$ der Medikamente in Tablettenform oder in Kapseln produziert [1].

In der Regel bestehen entsprechende Tabletten aus Pulver verschiedener Substanzen, die zusätzlich Wirkstoffe enthalten. Die Tabletten haben verschiedene physikalische Eigenschaften, abhängig von den verwendeten Komponenten (Partikelgröße, chemische Zusammensetzung) und dem Fertigungsprozess (Oberflächenform, Rauheit, Verdichtung und Porosität), welche die Qualität und die Wirkung beeinflussen. Aus diesem Grund könnte die Analyse physikalischer Parameter ein Weg sein, um die Qualität des Produktionsprozesses zu verbessern und zu kontrollieren [2, 3].

Es gibt verschiedene Techniken, um physikalische Eigenschaften von Tabletten $z u$ analysieren, wie beispielsweise die Nahinfrarotspektroskopie (NIR), die Rasterelektronenmikroskopie (REM) oder die energiedispersive Röntgenspektroskopie (EDX).

Eine dieser physikalischen Größen stellt die Rauheit dar. Da es sich bei der Rauheit um einen oberflächenspezifischen Parameter handelt, kann daraus auf die Qualität der Pressung oder auch die Reproduzierbarkeit des Fertigungsprozesses der Tabletten geschlossen werden. Außerdem beeinflusst die Rauheit auch den Schluckvorgang. Allgemein sind viele unterschiedliche Methoden bekannt, um die Rauheit einer Oberfläche zu bestimmen. Einerseits gibt es optische Verfahren (Weißlichtinterferometrie, Fokusvariation, Streulicht-Analyse, Streifenlichtprojektion, 
Parseval Theorem, Speckle-Elongation) $[4,5]$ und andererseits mechanische Verfahren (profilbasierte Methoden, wie z. B. das Tastschnittverfahren). Da für mechanische Messmethoden ein direkter Kontakt mit dem Messobjekt vorausgesetzt wird, ist für die Anwendung an Tabletten eine Beschränkung auf optische Verfahren sinnvoll. Eine kostengünstige, optische Messmethode stellt die Angulare Speckle Korrelation (ASK) dar. In dieser Arbeit wird die Bestimmung der Rauheit an ausgewählten Originalpräparaten von Tabletten und ihren Generika mittels ASK untersucht.

\section{Theorie der Speckle-Korrelation}

Es ist bekannt, dass durch die Korrelation zweier SpeckleMuster die Rauheit einer Oberfläche gemessen werden kann [6-8]. Im Bereich der Speckle-Techniken sind die Angulare Speckle-Korrelation (ASK) und die Spektrale Speckle-Korrelation (SSK) zwei der wichtigsten Techniken, die in der Forschung eingesetzt werden. Bei der ASK werden durch die Verwendung eines optischen Systems zwei unabhängige, separate Laserstrahlen unter einem bestimmten Winkel auf die raue Oberfläche fokussiert und die erzeugten Speckle-Muster in der Fourier-Ebene auf der CCD-Kamera aufgenommen. Bei der SSK ist die Methode ähnlich, aber anstelle der Variation des Beleuchtungswinkels bleibt der Strahlengang gleich und die Wellenlänge des Lasers wird verändert.

Unter der Annahme einer aus makroskopischer Sicht ebenen Fläche, einer gaußförmigen Oberflächenhöhenverteilung und bei Vernachlässigung von Mehrfachstreuungen und Schatteneffekten kann bei der ASK gezeigt werden $[6,7,9]$, dass sich der Korrelationskoeffizient $C$ aus beiden Speckle-Bildern durch die folgende Gleichung berechnen lässt:

$$
\begin{aligned}
& C\left(\alpha_{i}, \alpha_{0}, \delta \alpha, \Delta \xi, \Delta \eta\right) \\
& =\exp \left[-\left(\left(\sin \alpha_{i} \delta \alpha+\sin \alpha_{i} \frac{\Delta \xi}{f}\right) k \sigma_{h}\right)^{2}\right] \\
& \quad \cdot \exp \left[-\left(\frac{L k}{2 f}\right)^{2}\left(\left(\Delta \xi-\frac{\cos \alpha_{i}}{\cos \alpha_{0}} f \delta \alpha\right)^{2}+(\Delta \eta)^{2}\right)\right],
\end{aligned}
$$

wobei $\alpha_{0}$ der Beobachtungswinkel, $\alpha_{i}$ der Einfallswinkel, $\delta \alpha$ die Winkeldifferenz in der Beleuchtung, $f$ die Brennweite der Linse, $L$ der Abfall der Intensitätsverteilung im Laserbündel $\left(1 / e^{2}\right), k$ die Wellenzahl und $\sigma_{h}$ die Standardabweichung der Oberflächenhöhenverteilung sind. Die Verschiebungen $\Delta \xi$ und $\Delta \eta$ stellen aufgrund der Diffe- renz der Einfallswinkel beider Lichtstrahlen die Verschiebung des Speckle-Musters in der Fourier-Ebene dar. Durch Berechnen des Korrelationskoeffizienten kann $\sigma_{h}$ mit folgender Gleichung bestimmt werden:

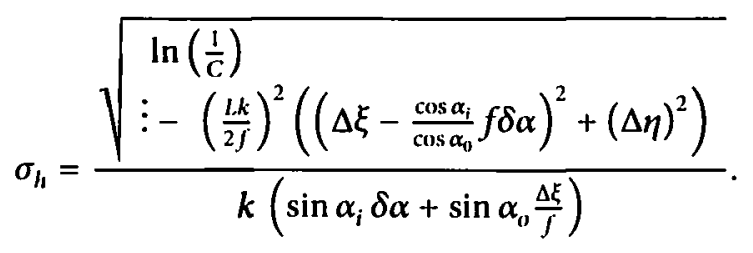

Obwohl Gl. (2) die Berechnung von $\sigma_{h}$ erlaubt, ist diese Umsetzung aus experimenteller Sichtweise nicht prakti kabel, weil sie von vielen Parametern abhängt und die systematischen Messfehler groß sind. Aus diesem Grund wird Gl. (2) vereinfacht, so dass die Fehler minimiert werden. Es kann einfach nachgewiesen werden, dass die opti malen Einstellungen den folgenden Bedingungen entsprechen $[6,7]$ :

$$
\Delta \xi-\frac{\cos \alpha_{i}}{\cos \alpha_{0}} f \delta \alpha=0, \quad \Delta \eta=0 .
$$

In diesem Fall sind lediglich Koordinatendifferenzen einzuhalten, die der durch die Einfallswinkeldifferenz $\delta \alpha$ verursachten homogenen Verschiebung der Speckle Bilder in der Ebene der CCD-Kamera entsprechen. Bei Berücksichtigung der Bedingungen der Gl. (3) ergibt sich für Gl. (2):

$$
\sigma_{h}=\frac{\sqrt{\ln \left(\frac{1}{\mathrm{c}}\right)}}{k\left(\sin \alpha_{i} \delta \alpha+\tan \alpha_{o} \cos \alpha_{i} \delta \alpha\right)} .
$$

Durch geometrische Optimierung der Anordnung kann der Einfallswinkel $\alpha_{i}$ und Beobachtungswinkel $\alpha_{0}$ gleich $\alpha$ ge

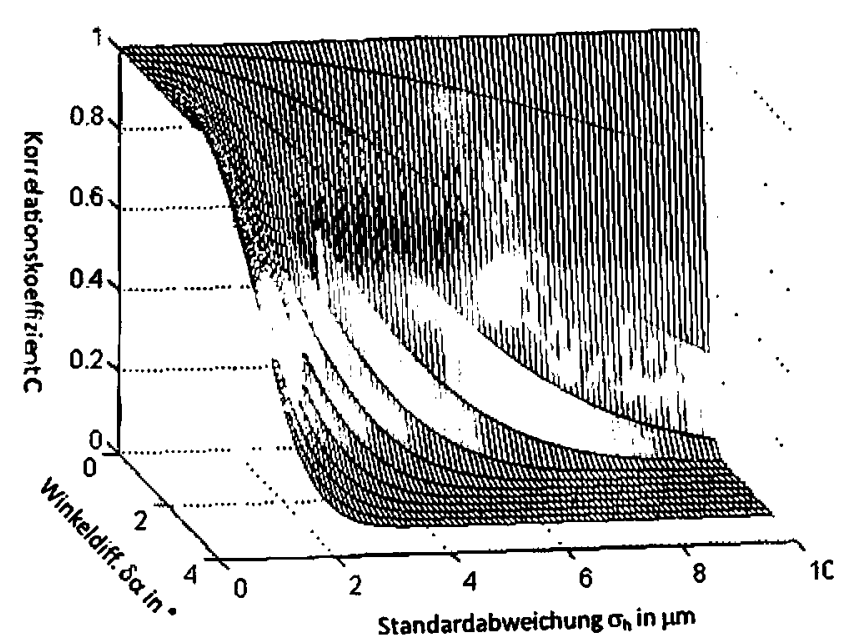

Abbildung 1: 3D-Darstellung des Korrelationskoeffizienten $C\left(\alpha=45^{\circ}\right.$ und $\lambda=632,8 \mathrm{~nm}$ ). 
setzt werden [10, 11]. Daraus ergibt sich aus Gl. (4) und $k=2 \pi / \lambda$ :

$$
\sigma_{h}=\frac{\lambda \sqrt{\ln \left(\frac{1}{c}\right)}}{4 \pi \delta \alpha \sin \alpha} .
$$

Abbildung 1 zeigt eine dreidimensionale Darstellung des Korrelationskoeffizienten $C$ als Funktion der Winkeldifferenz $\delta \alpha$ und der Standardabweichung $\sigma_{h}$. Für spezifische $\alpha$, $\delta \alpha$ und $\lambda$ ist $C\left(\sigma_{h}\right)$ nur von $\sigma_{h}$ abhängig und entspricht geometrisch einer der in Abbildung 1 dargestellten Kurven.

Mittels der Einstellung von $\delta \alpha$ kann der Messbereich des Speckle-Systems einfach an die Rauheit des Messobjekts angepasst werden.

\section{Experimentelles}

\subsection{Aufbau und Messverfahren}

In Abbildung 2 ist die experimentelle Anordnung des Speckle-Korrelationssystems dargestellt. Diese besteht aus einem Laser, einem Strahlteiler, einem Spiegel, zwei Shuttern, einer Linse, einer CCD-Kamera und einer Halterung mit der Probe. Der Beleuchtungsstrahl wird auf den Strahlteiler gerichtet, wo er sich in zwei Teilstrahlen aufteilt, die auf die gleiche Stelle der Probe (Messobjekt) fokussiert werden. Nach der korrekten Justierung beider Teilstrahlen wird der zweite Lichtpfad abgedeckt und das Experiment nur mit dem ersten Teilstrahl durchgeführt. Das von der Probe rückgestreute Licht wird durch die Linse mit der CCD Kamera aufgenommen und gespeichert. Mit der Zielsetzung Gl. (5) anzuwenden, muss die Kamera um $\Delta \xi=f \delta \alpha$ in der Ebene des CCD Chips verschoben und ei

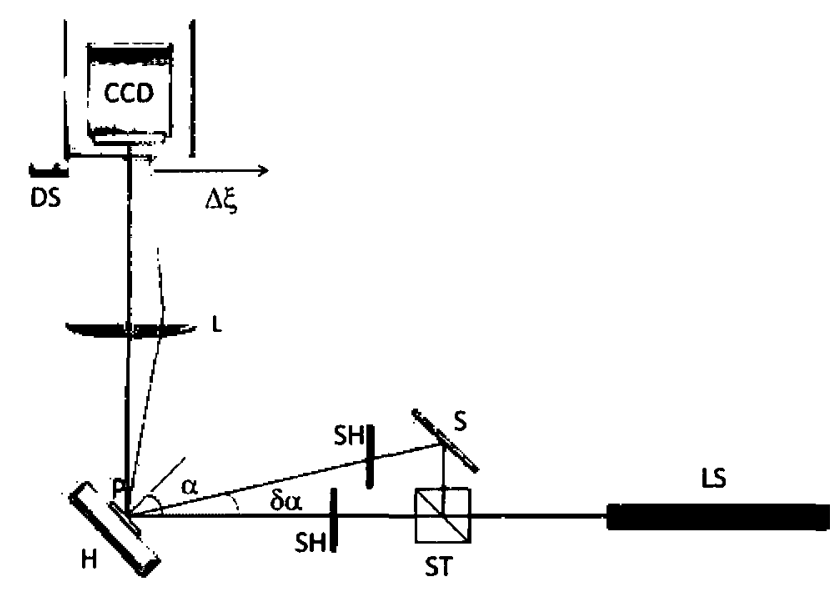

Abbildung 2: Experimenteller Aufbau zur ASK. LS: Laser, ST: Strahlteiler, S: Spiegel, SH: Shutter, H: Halterung, P: Probe, L: Linse, DS: Differentialmikrometerschraube, CCD: Kamera. ne zweite Aufnahme eines Speckle-Bildes aufgenommen werden, wenn der zweite Teilstrahl die Probe beleuchtet und der erste Teilstrahl abgedeckt ist. Der Korrelationskoeffizient $C$ entspricht dem Korrelationskoeffizienten der beiden Speckle-Bilder. Mit dieser Information und mittels Gl. (5) kann $\sigma_{h}$ der Probe berechnet werden. Da bei Anwendung der ASK an passenden Messoberflächen die Standardabweichung der Oberflächenhöhenverteilung $\sigma_{h}$ dem quadratischen Mittenrauheitswert $R_{q}$ entspricht, wird im Folgenden anstatt von $\sigma_{h}$ häufig von der Rauheit gesprochen.

Aufgrund der Ungenauigkeiten des Experiments, der Messfehler und beispielsweise der Schwingungen im Labor ist nicht gewährleistet, dass die Bedingungen aus Gl. (3) erfüllt werden. Anstatt die Kamera einmal um den festen Wert $f \delta \alpha$ zu verschieben und das Speckle-Bild mit dem zweiten Teilstrahl aufzunehmen, wird dagegen der Detektor schrittweise parallel zur Linsenebene verschoben und für jede Stellung eine Messung durchgeführt. Für jedes Bild dieser Aufnahmereihe wird der Korrelationskoeffizient mit dem ersten Speckle-Bild des ersten Teilstrahls berechnet. Auf diese Weise wird der maximale Korrelationskoeffizient und damit der Korrelationskoeffizient nach Gl. (5) bestimmt.

Um die Messzeit zu verkürzen, wird der Wert für $f \delta \alpha$ aus den Systemparametern berechnet und die Verschiebung somit um diesen Wert herum begrenzt. Um vergleichbare Ergebnisse zu erhalten, wird die Schrittweite für alle Messungen gleich gewählt, da diese Einfluss auf die Messgenauigkeit der Messwerte hat. Vorteil dieses Verfahrens ist, dass auch wenn die Randbedingungen nach Gl. (3) nicht exakt erfüllt werden, eine korrekte Positionierung der Kamera und somit eine präzise Rauheitsbestimmung im Rahmen der Messgenauigkeit durchgeführt werden kann $[10,11]$.

Durch Automatisierung der Kamerapositionierung kann das Messverfahren, z. B. für die Integration in einen Fertigungsprozess, bzgl. Messgenauigkeit und Messdauer optimiert werden. Alternativ kann der Maximalwert der Korrelation auch rein softwareseitig bestimmt werden bei Verwendung einer Kamera mit ausreichend größerer Pixelanzahl und gleichen Kennwerten (Pixelgröße und Empfindlichkeit) auch ohne Qualitätsverluste.

\subsection{Ergebnisse}

Mit dem Ziel, die Rauheit verschiedener Arten pharmazeutischer Tabletten mittels ASK zu vermessen, wird die Anordnung nach Abbildung 2 mit einem He-Ne Laser betrieben. Für das System wird eine kleine Winkel-differenz ge- 
wählt, damit die Messungen in einem großen Bereich der Rauheit durchgeführt werden können. Für den hier verwendeten Fall mit $\alpha=45^{\circ}, \lambda=632,8 \mathrm{~nm}$ und $\delta \alpha=0,8^{\circ}$ ergibt sich die mathematische Funktion des Korrelationskoeffizienten $C\left(\sigma_{h}\right)=\exp \left(-\left(0,2 \sigma_{h}\right)^{2}\right)$, wie in Abbildung 3 dargestellt.

Vergleichsmessungen an Tabletten mittels eines kommerziellen Weißlichtinterferometers können nicht verwendet werden, da aufgrund der Wölbung und des Rauheitsbereichs der Tabletten keine aussagekräftigen Messresultate erzielt werden können. Vergleichsmessungen mittels Tastschnittgerät liefern ebenfalls keine brauchbaren Ergebnisse, da die Tastspitze des Tastschnittgeräts die Oberfläche der Tabletten zerkratzt. Streulicht aus dem Inneren der Tablette hat bei der Untersuchung mittels ASK keinen messbaren, erkennbaren Einfluss auf die Rauheitsmessung und wird deshalb hier vernachlässigt.

Um die Funktionalität des ASK-Messaufbaus zu verifzieren, werden daher Vergleichsmessungen an ebenen Metallringen durchgeführt. An diesen Proben liefert sowohl die ASK als auch die Weißlichtinterferometrie eindeutige, übereinstimmende Ergebnisse. Durch Anpassung der lateralen Auflösung der beiden Messmethoden können die Abweichungen auf unter $4 \%$ beschränkt werden.

Die ASK-Messungen wurden an drei Tablettenarten, die den gleichen Wirkstoff, allerdings unterschiedliche Trägerstoffe enthalten, durchgeführt. Der Unterschied zwischen den beiden Originaltabletten und den Generika liegt prinzipiell nicht im Wirkstoff des Medikamentes, sondern in den Trägerkomponenten und der Effektivität der Wirkstoffe, wie beispielsweise der Zeit, die die Wirkstoffe im Blut bleiben oder wie schnell diese aus dem Körper

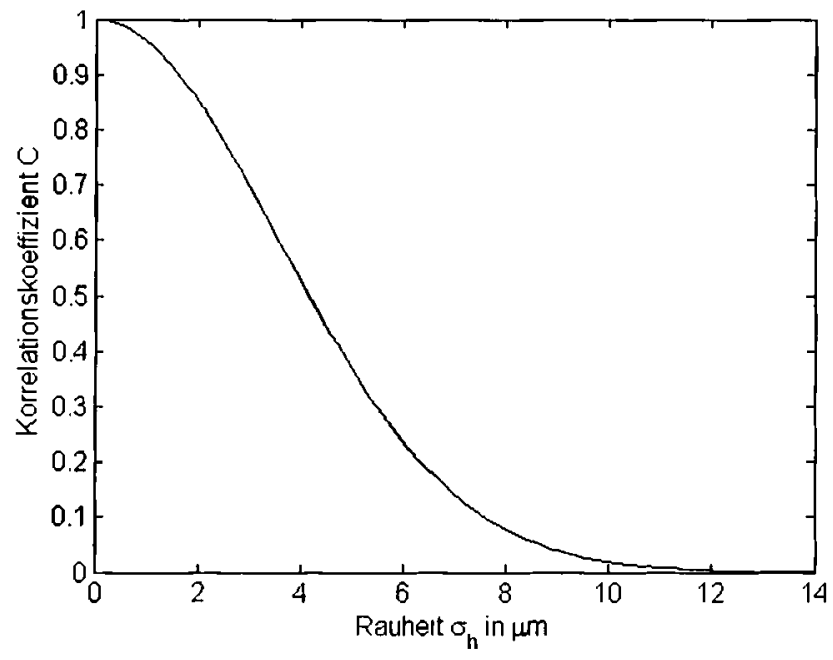

Abblldung 3: Korrelationsfunktion für $\lambda=632,8 \mathrm{~nm}, \alpha=45^{\circ}$ und $\delta \alpha=0,8^{\mathrm{R}}$.
Tabelle 1: Rauheitsmittelwerte der Messungen aus Abbildung 4 von Orginaltabletten und Generika mit gleichem Wirkstoff.

\begin{tabular}{lc}
\hline Tablette & Rauheit in $\mu \mathrm{m}$ \\
\hline Original (A) & 1,5 \\
Generikum 1 (B) & 1,3 \\
Generikum 2 (C) & 1,7 \\
\hline
\end{tabular}
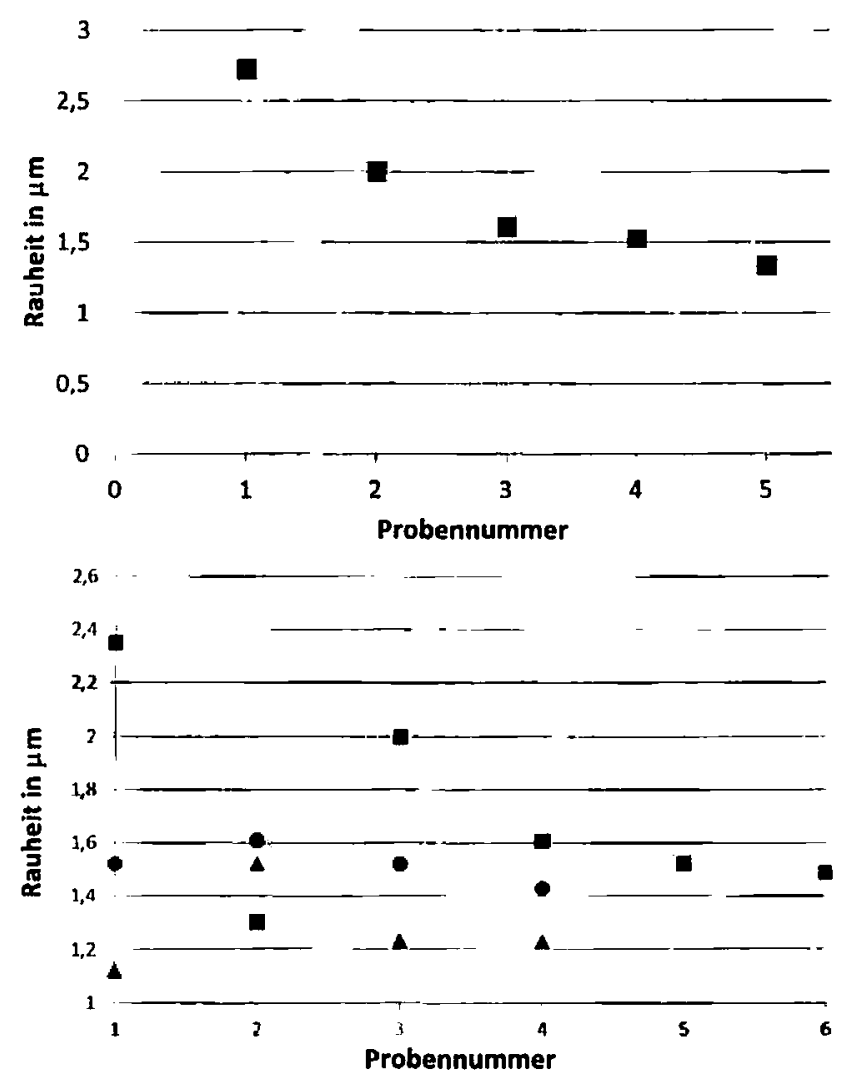

Abbildung 4: Alle gemessenen Rauheitswerte von Orginaltabletten ( $4 \times$ A: $\bullet$ und deren Generika ( $4 \times$ B: $\triangle ; 6 \times C: \square$ ).

wieder ausgeschieden werden. Neben den Trägerkompo nenten wirkt sich auch der jeweilige Produktionsprozess auf die Qualität des Endproduktes aus, was sich z. B. in der Oberflächenbeschaffenheit der Tabletten widerspiegelt.

Für die Messungen wird eine CCD-Kamera $(640 \times$ 480 Pixel) mit einer Pixelgröße von $6,7 \mu \mathrm{m} \times 6,7 \mu \mathrm{m}$ her angezogen. Der mittlere Speckle-Durchmesser beträgt ca. 5-15 Pixel.

In Tabelle 1 sind experimentell ermittelte Rauheits mittelwerte aufgezeigt. Die Differenzen zwischen den Rauheitsmittelwerten der einzelnen Produkte sind sehr gering und liegen im Bereich der systematischen Messfehler des Speckle-Systems, siehe Abschnitt 3.3. Deshalb kann prinzipiell keine definitive Schlussfolgerung aus den Ergebnissen gezogen werden. Trotzdem können brauchbare Rück- 
schlüsse aus den Ergebnissen isoliert werden, sobald eine separate Betrachtung der Messreihen stattfindet. Wie in Abbildung $4 \mathrm{zu}$ erkennen, schwanken die Rauheitswerte für die Tabletten Typ A und Typ B in der Größenordnung des systematischen Messfehlers, d. h., in diesen Fällen ist kein Fazit möglich. Die Rauheitswerte der einzelnen Tabletten vom Typ C liegen im Bereich von 1,3 $\mu \mathrm{m}$ bis 2,4 $\mu \mathrm{m}$, die auftretenden Abweichungen der Rauheitswerte mit bis $\mathrm{zu} 30 \%$ sind somit weitaus größer als systembedingte Messfehler. Diese enormen Schwankungen können durch eine Prozessoptimierung bzw. eine Prozessregelung mittels ASK reduziert werden.

Zusammenfassend kann aus den Rauheitswerten der Messreihen gefolgert werden, dass es sich bei der Her stellung der Tabletten (A) und (B) bereits um ein kontrolliertes, prozessoptimiertes Verfahren handelt. Eine wei tere Verbesserung ist mittels ASK nicht möglich, außer einer Kostenersparnis bei der Ablöse einer alternativen, teureren Messmethode. Für den Produktionsvorgang von Tabletten-Typ C könnte der Rauheitswert jedoch als Quali. tätsparameter zur Prozessregelung berücksichtigt werden.

\subsection{Betrachtung der Messfehler}

In diesem Abschnitt werden die systematischen Messfeh ler der Rauheitsmessung bestimmt. Zu diesem Zweck wird die Theorie der Unsicherheiten $[12,13]$ auf die Funktion $\sigma_{h}$ angewendet. Nach dieser Theorie kann für die Fortpflanzung der maximalen systematischen Messfehler geschrie ben werden:

$$
U_{s}(f)=\sum_{j}^{N}\left|\frac{\partial f}{\partial x_{j}}\right| U_{s}\left(x_{j}\right),
$$

wobei $x_{j}$ die verschiedenen, physikalisch messbaren Grö Ben der Gl. (5) darstellen und $U_{s}\left(x_{j}\right)$ die Einzelfehler jeder Variable sind. In dem hier vorliegenden Fall folgt daraus:

$$
f=\sigma_{h}(\lambda, \delta \alpha, \alpha)=\frac{\lambda \sqrt{-\ln C}}{4 \pi \delta \alpha \sin \alpha} .
$$

Aus den Gl. (6) und (7) ergibt sich

$$
\begin{aligned}
U_{s}\left(\sigma_{h}\right)= & \left|\frac{\sqrt{-\ln C}}{4 \pi \delta \alpha \sin \alpha}\right| U_{s}(\lambda) \\
& +\left|\frac{\lambda \cos \alpha \sqrt{-\ln C}}{4 \pi \delta \alpha(\sin \alpha)^{2}}\right| U_{s}(\alpha) \\
& +\left|\frac{\lambda \sqrt{-\ln C}}{4 \pi(\delta \alpha)^{2} \sin \alpha}\right| U_{s}(\delta \alpha)
\end{aligned}
$$

und der relative maximale systematische Messfehler berechnet sich aus

$$
\frac{U_{s}\left(\sigma_{h}\right)}{\sigma_{h}}=\left|\frac{U_{s}(\lambda)}{\lambda}\right|+\left|\frac{U_{s}(\alpha)}{\tan \alpha}\right|+\left|\frac{U_{s}(\delta \alpha)}{\delta \alpha}\right| .
$$

Die Messungen wurden unter folgenden Bedingungen durchgeführt: $U_{s}(\delta \alpha)=1,4 \times 10^{3} \mathrm{rad}\left(\approx 0,1^{10}\right), U_{s}(\lambda)=$ $0,1 \mathrm{~nm}$ und $U_{s}(\alpha)=1,7 \times 10^{2} \mathrm{rad}\left(1^{\circ}\right)$. Durch Einfügen dieser Werte in $\mathrm{Gl}$. (9) ergibt sich:

$$
\begin{aligned}
\frac{U_{s}\left(\sigma_{h}\right)}{\sigma_{h}} & =1,6 \times 10^{4}+1,7 \times 10^{-2}+8,4 \times 10^{2} \\
& =10,1 \% .
\end{aligned}
$$

Bei diesem Ergebnis ist der Einfluss jeder einzelnen Variablen der Gleichung (9) zu erkennen. Der dritte Term der Summe in Gl. (10) liefert den größten Wert und hängt von der Winkeldifferenz ab. Dies bedeutet, dass eine bessere Präzision bei der Messung von $\delta \alpha$ notwendig wird, um die Messwerte bei dieser Speckle-Methode zu verbessern. Allerdings ist dieser Term nicht nur deshalb wichtig, sondern auch, weil dieser Term direkt mit der Empfindlichkeit und dem Messbereich der ASK zusammen hängt.

\section{Zusammenfassung und Ausblick}

Eine vollständige Charakterisierung von pharmazeutischen Tabletten, die aus Pulver produziert werden, besteht nicht nur aus der chemischen Zusammensetzung, sondern auch aus dem Aufbau der inneren Struktur und der Oberflächenbeschaffenheit. In dieser Arbeit wurde die Rauheitsmessung an einem Originalpräparat und zwei Ge nerika mittels Angularer Speckle Korrelation (ASK) unter sucht, wobei nicht der Wirkstoff, sondern die Trägerkom ponenten und die Produktionsmethode den Unterschied zwischen Originalpräparat und Generika ausmachen.

Der Vorteil der Speckle-Messmethode gegenüber herkömmlichen Methoden besteht darin, dass nach der Jus tierung des Messaufbaus die Messungen berührungslos und kostengünstig durchgeführt werden können. Die Mes sergebnisse zeigen, dass die Mittelwerte der Rauheit für die drei untersuchten Tablettenarten ähnliche Werte auf weisen. Dennoch können sich die Rauheitswerte der Ta. bletten einer Produktionsserie deutlich vom Mittelwert unterscheiden, wie im Beispiel des billigsten Generikums gezeigt wurde.

Die Differenz zwischen dem Rauheitswert einer Tablette und dem Mittelwert könnte im Fall des billigsten Gene rikums als möglicher Qualitätsparameter verwendet werden, um die Regelung des Produktionsprozesses, z. B. in 
Bezug auf den Druck beim Pressen, zu verbessern. Die untersuchte ASK-Methode hat das Potenzial der SpeckleMesstechnik aufgezeigt, um den Produktionsprozess von Tabletten zu verbessern.

Um in-situ-Messungen mittels ASK zu ermöglichen, müsste der Einsatz von zwei Lasern mit unterschiedlichen Polarisationsrichtungen bei gleichzeitiger Detektion mit zwei Kameras untersucht werden. Eine weitere Alternative könnte die Speckle-Elongation [5] darstellen.

Die Formvermessung mittels Zwei-WellenlängenSpeckle-Interferometrie bietet den weiteren Vorteil, dass sie zusätzlich in-situ eingesetzt werden könnte, sofern die Speckle-Bilder gleichzeitig aufgenommen werden. Diese Methode wird in weiteren Forschungen um die aus der Spektroskopie bekannte multivariate Datenanalyse erweitert, um auch mit dieser Messtechnik Rauheits. parameter messen zu können [14]. Erste Ergebnisse sehen vielversprechend aus und die weitere Entwicklung der Speckle-Messtechnik zum Einsatz in der in-situ Rauheitsmessung bleibt abzuwarten.

\section{Literatur}

1. F.J. Muzzio, C. L. Goodridge, A. Alexander, P. Arratia, H. Yang, $O$. Sudah, and G. Mergen: Sampling and characterization of pharmaceutical powders and granular blends. In: International Journal of Pharmaceutics 250 (2003), S. 51-64.

2. P. Seitovuopio, I. Heinämäki, J. Rantanen, and J. Yliruusi: Monitoring Tablet Surface Roughness During the Film Coating Process. In: AAPS PharmSciTech 7 (2006), Nr. 2, S. E1-6.

Prof. Dr. Félix Salazar Bloise

ETSI Minas, C/Ríos Rosas 21, Departamento de Física Aplicada, 28003 Madrid, Spanien, Tel.: +34-91-336-4179, Fax: +34-91-3366952

felixjose.salazar@upm.es

Félix Salazar Bloise studierte von 1981 bis 1987 Physik an der Universidad Complutense de Madrid. Darauf folgte 1992 die Promotion mit Auszeichnung an der Universidad Politécnica de Madrid und anschließend ein Aufenthalt als Postdoc im Saarland (1994-1995). Seit 1995 hat er die Professur für "Angewandte Physik“ an der Fakultät für Bergbauingenieurswesen an der Universidad Politécnica de Madrid inne und forscht im Bereich der optischen und akustischen Messtechnik. Der Schwerpunkt liegt dabei in Anwendungen im Bereich der Verformungs- und Oberflächenanalyse sowie in der Charakterisierung von Flüssigkeiten.
3. S. Honary, H. Orafai: The Effect of Core Surface Tension on Roughness of Film Coated Tablets. In: Iranian Journal of Pharmaceutical Research (2003), S. 65- 66.

4. A. Duparré, J. Ferre-Borrull, S. Gliech, G. Notni, J. Steinert, and J. M. Bennett: Surface Characterization Techniques for Determining the Root-Mean-Square Roughness and Power Spectral Densities of Optical Components. In: Applied Optics 41 (2002), Nr. 1, S. 154-171.

5. P. Lehmann, S. Patzelt, and A. Schöne: Surface roughness measurement by means of polychromatic speckle elongation. In: Applied Optics 36 (1997), Nr. 10, S. 2188-2197.

6. B. Ruffing: Application of speckle-correlation methods to surface-roughness measurement: a theoretical study. In: I. Opt. Soc. Am. A (1986), Nr. 3, S. 1297-1304.

7. B. Ruffing: Berührungslose Rauheitsmessung technischer Oberflächen mit Speckle-Korrelationsverfahren. Dissertation, Universität Karlsruhe, 1987.

8. I. Yamaguchi, K. Kobayashi, L. Yaroslavsky: Measurement of surface roughness by speckle correlation. In: Opt. Eng. 43 (2004), S. 2753-2761.

9. A.W. Koch: Speckle Measurement Techniques for Surface Analysis. In: Speckle Photography and Speckle Interferometry and their Applications to Mechanic Solid Problems (2008), Editor F. Salazar.

10. F. Salazar and A. Barrientos: Surface Roughness Measurement on a Wing Aircraft by Speckle Correlation. In: Sensors 13 (2013), Nr. 9, S. 11772-11781.

11. F. Salazar, T. Belenguer, J. García, G. Ramos: On roughness measurement by angular speckle correlation. In: Metrol. Meas. Syst., 19 (2012), Nr. 2, 373-380.

12. J. R. Taylor: An introduction to error analysis. Mill Valey, University Science Books (1982).

13. E. Schrüfer: Elektrische Messtechnik, Hanser (2010).

14. T. Bodendorfer, M. Schardt, A. W. Koch: Quantitative surface roughness measurements using multivariate data analysis in speckle interferometry. In: Opt. Eng. 52(10) (Aug 13, 2013), S. 101917.

\section{M.Sc. Laura Aulbach}

Technische Universität München, Lehrstuhl für Messsystem und Sensortechnik, Arcisstraße 21, 80333 München, Deutschland, Tel.: +49-89-289-23353, Fax: +49-89-289-23348 l.aulbach@tum.de

Laura Aulbach ist wissenschaftliche Mitarbeiterin am Lehrstuhl für Messsystem- und Sensortechnik der Fakultät für Elektrotechnik und Informationstechnik an der Technischen Universität München. Ihr Schwerpunkt der Forschung liegt im Bereich der lasergestützten Oberflächenanalytik. 


\section{Dr.-Ing. Martin Jakobi}

Technische Universität München, Lehrstuhl für Messsystem und Sensortechnik, Arcisstraße 21, 80333 München, Deutschland,

Tel.: +49-89-289-23351, Fax: +49-89-289-23348

m.jakobi@tum.de

Martin Jakobi ist wissenschaftlicher Mitarbeiter am Lehrstuhl für Messsystem- und Sensortechnik der Fakultät für Elektrotechnik und Informationstechnik an der Technischen Universität München. Sein Schwerpunkt der Forschung liegt im Bereich der optomechatronischen Messsysteme.
Prof. Dr.-Ing. Dr. h. c. Alexander W. Koch

Technische Universität München, Lehrstuhl für Messsystem und Sensortechnik, Arcisstraße 21, 80333 München, Deutschland, Tel.: +49-89-289-23344, Fax: +49-89-289-23348 a.w.koch@tum.de

Alexander W. Koch leitet den Lehrstuhl für Messsystem- und Sensortechnik der Fakultät für Elektrotechnik und Informationstechnik an der Technischen Universität München. Ein Schwerpunkt der Forschung liegt im Bereich der optomechatronischen Messsysteme. 